\title{
The Relationship between Intracardiac Atrial Sensing Values and Atrial High-rate Episodes in Patients With Permanent Pacemakers Implanted due to Sick Sinus Syndrome
}

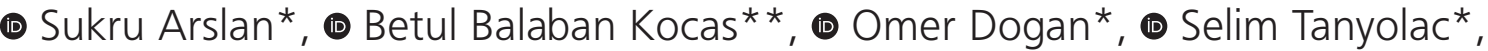 \\ (1) Isil Uzunhasan* \\ *Cardiology Institute of Istanbul University-Cerrahpasa, Department of Cardiology, Istanbul, Turkey \\ **Istanbul Prof. Dr. Cemil Tascioglu City Hospital, Clinic of Cardiology, Istanbul, Turkey
}

\section{Abstract}

\begin{abstract}
Aim: An increase in fibrosis and collagen in the atrium and sinus node tissues constitutes the basic pathophysiology of sick sinus syndrome (SSS). We aimed to investigate the relationship between P-wave sensing values and atrial high rate episodes (AHRE) in patients with dual-chamber (DDDR) pacemakers implanted due to SSS.

Methods: Patients with DDDR pacemakers implanted due to SSS between 2011 and 2019 were included in our retrospective cohort study. The patients were called for a visit and pacemaker controls were performed. The study population was divided into two groups according to the development of AHRE.

Results: At least one AHRE episode was detected in 51.7\% of participants. Left atrial (LA) diameters were increased in the AHRE+ group $(p<0.01)$. P-wave sensing values were significantly lower in the AHRE+ group $(p=0.01)$. LA diameter [hazard ratio $(H R)$ : 1.10 , 95\% (1.04-1.18), $p<0.01$ ] and P-wave sensing values [HR: 0.74, confidence interval 95\% $(0.57-0.95) p=0.02$ ] were determined as independent predictors of AHRE.
\end{abstract}

Conclusion: Our study demonstrated that LA diameter and P-wave sensing values were found to be independent predictors of AHRE development. Lower initial P-wave sensing values seem to be an important risk factor for AHRE development in this patient group.

Keywords: Sinoatrial node, permanent pacemakers, cardiac arrhythmias

\section{Introduction}

Sick sinus syndrome (SSS) is an important clinical condition presenting as isolated sinus bradycardia, sinusal arrest, sinus bradycardia and atrial arrhythmias. An increased amount of collagen and fibrosis in the sinoatrial node and atrium tissue constitutes the basic pathophysiology of SSS $(1,2)$. Most studies reported that increased fibrosis in the atrial tissue may both cause prolongation of atrial conduction time and increase the frequency of atrial arrhythmias $(3,4)$. In addition, some studies have reported that low atrial sensing values may be associated with increased volume load and scarring in the atrial tissue, thereby causing atrial arrhythmias (4-6).
Dual-chamber (DDDR) pacemaker implantation is the main treatment strategy to improve symptoms in this patient group. Although permanent pacemakers provide improvement in symptoms in SSS, their impact on mortality is still controversial $(1,7,8)$. Since these patients are mostly of advanced age, it is important to follow them in terms of atrial arrhythmias (9). Therefore, we aimed to investigate the relationship between the initial intracardiac P-wave sensing values and the development of atrial high rate episodes (AHRE) in patients with DDDR permanent pacemakers implanted due to SSS. 


\section{Methods}

\section{Ethical Approval and Study Population}

All patients gave their written informed consent, and the study was approved by the local ethics committee (Ethics Committee of Cardiology Institute of Istanbul University-Cerrahpasa, 2.i.Ü.E.50.0.05.00/4). Patients with DDDR pacemakers implanted due to SSS between 2011 and 2019 were included in our single-center retrospective cohort study. Patients with pacemaker implantation for atrioventricular node disease, non-optimal atrial lead location, ejection fraction (EF) $<40 \%$, persistent or permanent atrial fibrillation (AF), patients with implantable cardioverter-defibrillator (ICD), severe organ failure (chronic liver, chronic renal failure, etc.), active malignancy, prior radiotherapy, and systemic rheumatic disease were excluded from the study. A total of 118 patients were analyzed after excluding the patients with one of the exclusion criteria and those who could not be reached. The flow chart of the study is given in Figure 1.

\section{Study Protocol}

SSS is defined for patients who present with syncope, presyncope, effort dyspnea, fatigue, or palpitation with no other underlying cause, and sinus bradycardia, sinusal arrest, sinoatrial block, and/or accompanying atrial arrhythmias are detected in the examinations (10). Initial demographic characteristics, laboratory parameters and baseline pacemaker measurements of the study group were recorded by the electronic hospital system. In addition, the atrial lead position of the patients was examined from the hospital database; the right atrium appendix and the anterior and lateral areas close to this region were determined as the optimal lead locations. Only the data of patients whose recent pacemaker control data and event records were available in the hospital system for the last 6 months were recorded.

Patients with no recent data were called for a control visit, pacemaker measurements were performed, and events were recorded. Pacemaker controls were performed by two experienced physicians. Patients with AHRE episodes that exceeded 175/min and lasted longer than 30 seconds were included in the AHRE (+) group (11). Patients who did not have AHRE episodes or whose attacks were less than the specified criteria constituted the AHRE (-) group.

\section{Clinical Outcomes}

The primary endpoints of the study were defined as death, myocardial infarction, cerebrovascular events, and

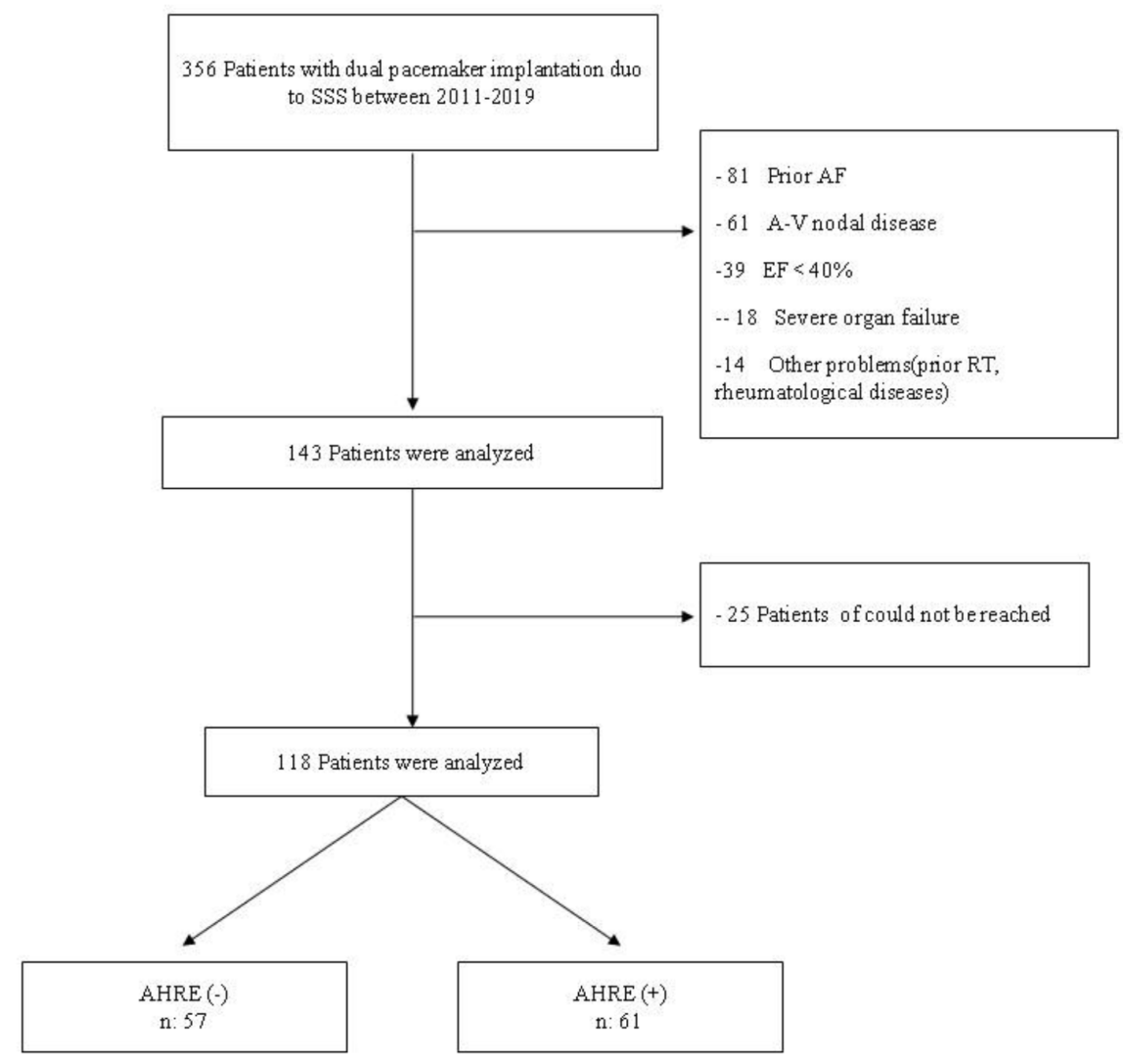

Figure 1. Trial flow diagram

AHRE: Atrial high rate episodes, AF: Atrial fibrillation, EF: Ejection fraction, SSS: Sick sinus syndrome 
pacemaker-related complications. The information related to the primary endpoints was obtained from the hospital database and patient visits.

\section{Statistical Analysis}

The SPSS version 21.0 (SPSS Inc., Chicago, Illinois) packet program was used for analysis. Normal distribution analysis of the data was performed by analytical methods such as the Kolmogorov-Smirnov and Shapiro-Wilks tests. Continuous variables were presented as means followed by standard deviation, and categorical variables as frequency and percentage. Continuous variables between the AHRE $(+)$ and AHRE (-) groups were compared using Student's t-tests for normally distributed data and the Mann-Whitney $U$ test for abnormally distributed data. Categorical parameters were evaluated by Pearson's chi-square test. A model of AHRE predictors was devised using Cox proportional multivariable analysis. A p-value $<0.05$ (2 tailed) was considered statistically significant.

\section{Results}

\section{Clinical and Demographical Features and Laboratory Parameters}

An AHRE was developed in $51.7 \%$ of the study group during a mean follow-up time of $49.4 \pm 8$ months. The mean age of the patients was $57.9 \pm 16$ years. In addition, $54.2 \%$ of the patients were female. The mean age of the AHRE+ group was significantly higher $(p=0.03)$. From the initial echocardiographic parameters, left atrium (LA) and right ventricular enddiastolic (Rvd) diameters were significantly higher in the AHRE+ group $(p<0.01$ and $p=0.04$; respectively). Tricuspid annular plane systolic excursion (TAPSE) values were significantly lower in the AHRE+ group $(p<0.01)$. In addition, alanine transaminase, leukocyte, total cholesterol, and low-density lipoprotein cholesterol levels were significantly lower in the AHRE+ group $(p<0.01, p<0.01, p=0.02, p<0.01$, respectively). According to the initial pacemaker and lead parameters, P-wave sensing values were significantly lower in the AHRE+ group $(p=0.01)$. Atrial and ventricular pacing rates, lead impedances and atrial threshold levels were similar between the groups $(p=0.64, p=0.52, p=0.44$, $p=0.31$, respectively). Demographic and biochemical parameters of the groups were given in detail in Tables 1 and 2.

\section{Clinical Outcomes}

The rates of primary outcomes, such as death, myocardial infarction, cerebrovascular accident, and pacemaker-related complications, at a mean follow-up of $49.4 \pm 8$ months were given in Table 3. Primary outcomes were significantly higher in the AHRE+ group $(p<0.01)$.

\section{Multivariable Analysis}

Predictors of AHRE were determined by a coxregression multivariable analysis model (Table 4). This model consisted of age, diabetes mellitus, LA, left ventricular end-diastolic diamater and Rvd, TAPSE, leukocyte and P-wave sensing values. LA diameter [hazard ratio (HR): 1.10, confidence interval $(\mathrm{Cl}) 95 \%(1.04-1.18), p<0.01]$ and P-wave sensing value [HR: 0.74, Cl 95\% (0.57-0.95), $\mathrm{p}=0.02$ ] were determined as independent predictors for the development of AHRE.

\section{Discussion}

Our results demonstrated that initial LA and RVd diameters were significantly higher in the AHRE+ group. Initial P-wave sensing values were lower in the positive AHRE group, but there was no significant difference in atrial and ventricular pacing rates. In addition, LA diameter and $P$ wave sensing values were defined as independent predictors of AHRE development.

SSS is a clinical condition that mostly occurs at advanced ages (2). Degeneration caused by increased fibrosis as a result of ischemia, collagen and fat deposition in the sinoatrial node is the most common cause of SSS $(1,12,13)$. Likewise, similar changes are expected to occur in the atrium. It has been reported that these changes cause deterioration of electrical homogeneity and prolongation of conduction times in the atrium that may lead to atrial arrhythmias (14-17). Also, increased fibrosis in the atrial tissue may also decrease the electrical activity of the atrium. The hypothesis that initial P-wave sensing values obtained from the atrial lead in sinus rhythm may be predictive of AHRE, especially in patients with permanent pacemakers, formed the basis of our study. In a cohort study of patients with low EF and an ICD, low atrial sensing values were associated with atrial arrhythmias. It has been documented that initial atrial sensing values below $1.5 \mathrm{mV}$ are a strong predictor of AHRE (5). Nielsen et al. (4) showed that an initial long P-Q interval predicts atrial arrhythmias in patients with DDDR pacemakers implanted due to HSS. In addition, they found lower atrial sensing values in patients with AF. The main difference between this study and ours was the inclusion of patients with AF. Healey et al. (18) reported that LA diameter and LA volume were defined as the predictors of AHRE. Similarly, Mathen and Chase (19) and Wu et al. (20) demonstrated that LA diameter was higher in the AHRE+ group and LA diameter was found as an independent predictor of AHRE. Unlike in these studies, in a study conducted with 109 patients with permanent DDDR pacemakers implanted for SSS, there was no relationship between P-wave interval and P-wave dispersion and atrial arrhythmias (21). Conversely, some studies demonstrated that prolonged 


\begin{tabular}{|c|c|c|c|}
\hline & \begin{tabular}{|l|} 
AHRE (-) \\
$(n=57)$
\end{tabular} & $\begin{array}{l}\text { AHRE (+) } \\
(\mathrm{n}=61)\end{array}$ & p-value \\
\hline Age (years) & $54.9 \pm 15$ & $60.8 \pm 17$ & $0.025^{b}$ \\
\hline Female, n (\%) & $33(57.9 \%)$ & $31(50.8 \%)$ & 0.441 \\
\hline Hypertension, n (\%) & $24(42.1 \%)$ & $31(50.8 \%)$ & 0.343 \\
\hline Diabetes mellitus, n (\%) & $13(22.8 \%)$ & $13(21.3 \%)$ & 0.850 \\
\hline Hyperlipidemia, n (\%) & $12(21.1 \%)$ & $12(19.7 \%)$ & 0.852 \\
\hline Active smoking, $\mathrm{n}(\%)$ & $23(40.4 \%)$ & $31(50.8 \%)$ & 0.254 \\
\hline Previous MI, n (\%) & $5(8.8 \%)$ & $5(8.2 \%)$ & 0.911 \\
\hline Previous CABG, $n(\%)$ & $7(12.3 \%)$ & $5(8.2 \%)$ & 0.463 \\
\hline $\mathrm{BB}, \mathrm{n}(\%)$ & $17(29.8 \%)$ & $23(37.7 \%)$ & 0.366 \\
\hline$C C B, n(\%)$ & $6(10.5 \%)$ & $10(16.4 \%)$ & 0.352 \\
\hline Amiodarone, n (\%) & 0 & $4(6.6 \%)$ & * \\
\hline Propafenon, n (\%) & $10(17.5 \%)$ & $15(24.6 \%)$ & 0.349 \\
\hline Statin, n (\%) & $15(26.3 \%)$ & $18(29.5 \%)$ & 0.699 \\
\hline LVEF \% & $59.3 \pm 2$ & $59.6 \pm 2$ & 0.450 \\
\hline $\mathrm{LA}(\mathrm{mm})$ & $35.6 \pm 6$ & $39.9 \pm 5$ & $<0.001^{c}$ \\
\hline $\mathrm{LVd}(\mathrm{mm})$ & $47.6 \pm 5$ & $48.5 \pm 5$ & 0.256 \\
\hline $\mathrm{RVd}(\mathrm{mm})$ & $22.8 \pm 3$ & $23.6 \pm 2$ & $0.044^{c}$ \\
\hline IVS (mm) & $9.8 \pm 1$ & $10.3 \pm 1$ & 0.116 \\
\hline TAPSE $(\mathrm{mm})$ & $23.7 \pm 3$ & $22.1 \pm 3$ & $0.002^{c}$ \\
\hline \multicolumn{4}{|c|}{ Initial lead measurements } \\
\hline P-wave sensing $(\mathrm{mV})$ & $3.07 \pm 1.4$ & $2.40 \pm 1.1$ & $0.016^{c}$ \\
\hline Atrial threshold $(\mathrm{V})$ & $0.8 \pm 0.2$ & $0.8 \pm 0.3$ & 0.310 \\
\hline Atrial impedance (ohm) & $627.6 \pm 176$ & $594.3 \pm 139$ & 0.489 \\
\hline Total AP, \% & $69.4 \pm 23$ & $63.3 \pm 29$ & 0.635 \\
\hline Total VP, \% ${ }^{\mathrm{a}}$ & $8(1-99)$ & $10(1-100)$ & 0.519 \\
\hline Follow-up time (month) & $49.8 \pm 8$ & $48.9 \pm 8$ & 0.648 \\
\hline \multicolumn{4}{|c|}{$\begin{array}{l}\text { a: Median (min-max), b:Mann-Whitney U test, c: Student's t-test } \\
\text { The mean age and LA diameter were considerably larger in the AHRE+ group, whereas the TAPSE and P wave sensing values were significantly lower. } \\
\text { AP: Atrial pacing, BB: Beta blocker, CCB: Calcium channal blocker, CABG: Coronary artery bypass grafting, LA: Left atrium, LVd: Left ventricular diastolic diamater, RVd: } \\
\text { Right ventricular diastolic diameter, IVS: Interventricular septum thickness, TAPSE: Tricuspid annular plane systolic excursion, LVEF: Left ventricular ejection fraction, MI: } \\
\text { Myocardial infarction, VP: Ventricular pacing, AHRE: Atrial high rate episode }\end{array}$} \\
\hline
\end{tabular}

P-wave duration before pacemaker implantation was an independent predictor of worsening atrial fibrillation burden after pacemaker implantation $(22,23)$.

Likewise, LA diameter was higher in the AHRE+ group, and it was found to be an independent predictor of AHRE. In addition, atrial lead sensing values in initial sinus rhythm were found to be predictive of AHRE in patients with DDDR pacemaker implanted due to SSS. No other study regarding this subject was encountered in the literature. In this respect, we believe that our study may contribute to the source data.

\section{Study Limitations}

There were some limitations to be reported in our study. First of all, it was a single-center, retrospective study, and the number of patients was relatively low. Secondly, because of the retrospective nature of the study, we could not obtain the initial electrocardiograms. Despite these limitations, evaluation of the lead positions and pacemaker controls by two independent physicians, and obtaining records from cardiac devices, thereby providing subclinical atrial arrhythmias detection, were the superior aspects of our study.

\section{Conclusion}

Our results showed that higher initial LA and RVd diameters were reported in patients with DDDR pacemakers implanted due to SSS and who developed AHRE during follow-up. Also, initial P-wave sensing values were lower in patients who developed AHRE. Baseline LA diameter and P-wave sensing values were found to be independent predictors of AHRE development. In conclusion, initial low P-wave sensing values may be considered as a risk factor 
Table 2. Baseline biochemical characteristics of study

\begin{tabular}{|c|c|c|c|}
\hline & $\begin{array}{l}\text { AHRE (-) } \\
(n=57)\end{array}$ & $\begin{array}{l}\text { AHRE }(+) \\
(n=61)\end{array}$ & p-value \\
\hline Glucose (mg/dL) & $97.2 \pm 18$ & $97.9 \pm 16$ & 0.716 \\
\hline HbA1c (\%) & $5.8 \pm 1$ & $5.9 \pm 1$ & 0.240 \\
\hline Kreatinine (mg/dL) & $0.8 \pm 0.2$ & $0.9 \pm 0.2$ & 0.392 \\
\hline $\mathrm{ALT}^{*}$ & $20(7-89)$ & $15(5-33)$ & $0.003^{a}$ \\
\hline Hemoglobin (mg/dL) & $12.9 \pm 1.3$ & $13.2 \pm 0.7$ & 0.571 \\
\hline Leukocytes $\left(/ \mathrm{mm}^{3}\right)$ & $7629.8 \pm 2103$ & $6681.9 \pm 1735$ & $0.009^{b}$ \\
\hline Lymphocytes $\left(/ \mathrm{mm}^{3}\right)$ & $1989.5 \pm 1030$ & $1898.4 \pm 597$ & 0.771 \\
\hline Platelets $\left(10^{3} / \mathrm{mm}^{3}\right)$ & $223.9 \pm 8$ & $218.7 \pm 65$ & 0.436 \\
\hline Total cholesterol (mg/dL) & $196.3 \pm 41$ & $181.3 \pm 35$ & $0.020^{b}$ \\
\hline LDL cholesterol (mg/dL) & $135.1 \pm 38$ & $118.7 \pm 33$ & $0.006^{b}$ \\
\hline HDL cholesterol (mg/dL) & $50.5 \pm 13$ & $52.2 \pm 14$ & 0.509 \\
\hline Triglycerides (mg/dL) & $125.1 \pm 39$ & $112.9 \pm 52$ & 0.068 \\
\hline $\mathrm{CRP}$ * & $6(1-28)$ & $5(1-48)$ & 0.273 \\
\hline
\end{tabular}

*Median (min-max), a:Mann-Whitney U Test, b: Student's t-test.

ALT, leukocyte, total cholesterol and LDL cholesterol levels were significantly lower in the AHRE+ group.

ALT: Alanine aminotransferase, CRP: C-reactive protein, HbA1c: Hemoglobin A1C, HDL: High-density lipoprotein, LDL: Low-density lipoprotein, AHRE: Atrial high rate episode

\begin{tabular}{|l|l|l|l|}
\hline \multicolumn{5}{|l|}{ Table 3. Clinical events at long-term follow-up } \\
\hline & $\begin{array}{l}\text { AHRE (-) } \\
(\mathbf{n}=57)\end{array}$ & $\begin{array}{l}\text { AHRE (+) } \\
(\mathbf{n}=61)\end{array}$ & p-value \\
\hline Death, $\mathrm{n}(\%)$ & 0 & $5(8.2 \%)$ & * \\
\hline MI, n (\%) & 0 & 0 & * \\
\hline CVA, n (\%) & 0 & $7(11.5 \%)$ & * \\
\hline PAC, n (\%) & $1(1.8 \%)$ & 0 & * \\
\hline All events & $1(1.8 \%)$ & $12(19.7 \%)$ & $\mathbf{0 . 0 0 2}$ \\
\hline $\begin{array}{l}\text { a: Chi-square test. } \\
\text { Primary outcomes were significantly higher in the AHRE+ group. } \\
\text { CVA: Cerebrovascular accident, MI: Myocardial infarction, PAC: Pacemaker } \\
\text { associated complication, AHRE: Atrial high rate episode }\end{array}$ \\
\hline
\end{tabular}

\section{Table 4. Multivariable predictors of AHRE occurance}

\begin{tabular}{|l|l|l|l|}
\hline & HR & \%95 Cl & p-value \\
\hline Age & 1.01 & $(0.99-1.03)$ & 0.281 \\
\hline DM & 0.85 & $(0.43-1.71)$ & 0.652 \\
\hline LA & 1.10 & $(1,04-1,18)$ & $\mathbf{0 . 0 0 3}^{\mathrm{a}}$ \\
\hline LVd & 0.94 & $(0.88-1,01)$ & 0.059 \\
\hline RVd & 1.05 & $(0.91-1.22)$ & 0.504 \\
\hline TAPSE & 0.95 & $(0.88-1.03)$ & 0.217 \\
\hline WBC & 0.99 & $(0.97-1.03)$ & 0.107 \\
\hline P-wave sensing & 0.74 & $(0.57-0.95)$ & $\mathbf{0 . 0 2 0 ^ { a }}$ \\
\hline
\end{tabular}

a: Cox proportional multivariable analysis.

Analysis Model: Omnibus Test Model-2 likelihood: 482,3; Chi-square 28,8; $p<0,001$.

LA diameter and P-wave sensing value were determined as independent predictors for development of AHRE.

Cl: Confidence interval, DM: Diabetes mellitus, LA: Left atrium, LVd: Left ventricular end-diastolic diamater, RVd: Right ventricular end-diastolic diamater, TAPSE: Tricuspid annular plane systolic excursion, WBC: White blood cell, HR: Hazard ratio, AHRE: Atrial high rate episode for AHRE development in patients with DDDR pacemakers implanted due to SSS. In this context, further prospective studies with a large number of patients are warranted.

\section{Ethics}

Ethics Committee Approval: The study was approved by the local ethics committee (Ethics Committee of Cardiology Institute of Istanbul University-Cerrahpasa 2.i.ü.E.50.0.05.00/4).

Informed Consent: All patients gave their written informed consent.

\section{Authorship Contributions}

Concept: S.A., Design: S.A., O.D., Data Collection or Processing: S.T., Analysis or Interpretation: O.D., Literature Research: S.A., I.U., Writing: S.A., B.B.K.

Conflict of Interest: No conflict of interest was declared by the authors.

Financial Disclosure: The authors declared that this study received no financial support.

\section{References}

1. Wahls SA. Sick sinus syndrome. Am Fam Physician 1985;31:117-24.

2. Lamas GA, Lee $K$, Sweeney $M$, et al. The mode selection trial (MOST) in sinus node dysfunction: design, rationale, and baseline characteristics of the first 1000 patients. Am Heart J 2000;140:541-51.

3. Schwartzman D, Chang I, Michele JJ, Mirotznik MS, Foster KR. Electrical impedance properties of normal and chronically infarcted left ventricular myocardium. J Interv Card Electrophysiol 1999;3:213-24. 
4. Nielsen JC, Thomsen PE, Højberg S, et al. Atrial fibrillation in patients with sick sinus syndrome: the association with PQ-interval and percentage of ventricular pacing. Europace 2012;14:682-9.

5. Zecchin M, Solimene F, D'Onofrio A, et al. Atrial signal amplitude predicts atrial high-rate episodes in implantable cardioverter defibrillator patients: Insights from a large database of remote monitoring transmissions. J Arrhythm 2020;36:353-62.

6. Li C, Ju W, Gu K, et al. Atrial involvement and progression of sinus node dysfunction in non-senile patients: evidences from electroanatomic mapping and long-term follow-up. J Interv Card Electrophysiol 2021;60:365-73.

7. Andersen HR, Nielsen JC, Thomsen PE, et al. Long-term follow-up of patients from a randomised trial of atrial versus ventricular pacing for sick-sinus syndrome. Lancet 1997;350:1210-6.

8. McComb JM, Gribbin GM. Effect of pacing mode on morbidity and mortality: update of clinical pacing trials. Am J Cardiol 1999;83:211-3.

9. Yang PS, Kim D, Jang E, et al. Risk of sick sinus syndrome in patients diagnosed with atrial fibrillation: A population-based cohort. J Cardiovasc Electrophysiol 2021;32:2704-14.

10. AdanV, Crown LA. Diagnosis and treatment of sick sinus syndrome. Am Fam Physician 2003;67:1725-32.

11. Hindricks G, Potpara T, Dagres N, et al. 2020 ESC Guidelines for the diagnosis and management of atrial fibrillation developed in collaboration with the European Association for CardioThoracic Surgery (EACTS): The Task Force for the diagnosis and management of atrial fibrillation of the European Society of Cardiology (ESC) Developed with the special contribution of the European Heart Rhythm Association (EHRA) of the ESC. Eur Heart J 2021;42:373-498.

12. Rodriguez RD, Schocken DD. Update on sick sinus syndrome, a cardiac disorder of aging. Geriatrics 1990;45:33-6.

13. Tierney LM, McPhee SJ, Papadakis MA. Heart conduction disturbances: sick sinus syndrome. In: Papadakis MA, Tierney LM, McPhee SJ, editors. Current medical diagnosis
\& treatment, 39th ed. New York, N.Y: Lange Medical Books/ McGraw-Hill; 2000. p. 412.

14. Brachmann J, Karolyi L, Kübler W. Atrial dispersion of refractoriness. J Cardiovasc Electrophysiol 1998;9(Suppl 8):35-9.

15. Liu Z, Hayano M, Hirata $T$, et al. Abnormalities of electrocardiographic $\mathrm{P}$ wave morphology and their relation to electrophysiological parameters of the atrium in patients with sick sinus syndrome. Pacing Clin Electrophysiol 1998;21:79-86.

16. Centurion OA, Isomoto S, Shimizu A, et al. Supernormal atrial conduction and its relation to atrial vulnerability and atrial fibrillation in patients with sick sinus syndrome and paroxysmal atrial fibrillation. Am Heart J 1994;128:88-95.

17. Centurion OA, Isomoto S, Fukatani M, et al. Relationship between atrial conduction defects and fractionated atrial endocardial electrograms in patients with sick sinus syndrome. Pacing Clin Electrophysiol 1993;16:2022-33.

18. Healey JS, Martin JL, Duncan A, et al. Pacemaker-detected atrial fibrillation inpatients with pacemakers: prevalence, predictors, and current use of oral anticoagulation. Can J Cardiol 2013;29:224-8.

19. Mathen PG, Chase D. Pacemaker detected prolonged atrial high rate episodes - Incidence, predictors and implications; a retrospective observational study. J Saudi Heart Assoc 2020;32:157-65.

20. Wu Z, Chen X, Ge J, Su Y. The risk factors of new-onset atrial fibrillation after pacemaker implantation. Herz 2021;46(Suppl 1):61-8.

21. Kristensen L, Nielsen JC, Mortensen PT, et al. Sinus and paced P wave duration and dispersion as predictors of atrial fibrillation after pacemaker implantation in patients with isolated sick sinus syndrome. Pacing Clin Electrophysiol 2004;27:606-14.

22. Murase $Y$, Imai $H$, Ogawa $Y$, et al. Usefulness of P-wave duration in patients with sick sinus syndrome as a predictor of atrial fibrillation. J Arrhythm 2021;37:1220-6.

23. Nishinarita R, Niwano S, Oikawa J, et al. Novel Predictor for New-Onset Atrial High-Rate Episode in Patients With a DualChamber Pacemaker. Circ Rep 2021;3:497-503. 